\title{
Current Problems of Diagnostics and Treatment of Purulent-Inflammatory Diseases and Sepsis in Medical Practice
}

\author{
Lesia Ostapiuk $^{1 *}$, Anatolii Voloshinovskii ${ }^{2}$, Vasyl Savchyn ${ }^{3}$, Nataliia Tuziyk ${ }^{3}$, Taras Malui \\ ${ }^{1}$ Lviv Regional Public Health Centre, Lviv, Ukraine \\ ${ }^{2}$ Department of Experimental Physics, Ivan Franko Lviv National University, Lviv, Ukraine \\ ${ }^{3}$ Communal Clinical Hospital No. 8, Lviv, Ukraine \\ Email: ^lesya_ost@ukr.net
}

How to cite this paper: Ostapiuk, L., Voloshinovskii, A., Savchyn, V., Tuziyk, N. and Malui, T. (2021) Current Problems of Diagnostics and Treatment of PurulentInflammatory Diseases and Sepsis in Medical Practice. International Journal of Clinical Medicine, 12, 87-107.

https://doi.org/10.4236/ijcm.2021.123011

Received: February 12, 2021

Accepted: March 19, 2021

Published: March 22, 2021

Copyright $\odot 2021$ by author(s) and Scientific Research Publishing Inc. This work is licensed under the Creative Commons Attribution International License (CC BY 4.0).

http://creativecommons.org/licenses/by/4.0/

\begin{abstract}
Background: Poor treatment of burns can lead to sepsis and even death. Especially promising is the use of the method of fluorescence spectroscopy for its diagnostics. The aim of the research is to create a pathogenetic concept as the basis of a diagnostic and treatment model of purulent-inflammatory diseases and sepsis. Material and methods: The study lasted from 2001 to 2019 and included 4 stages. The experimental base of luminescent research at all stages was the luminescence laboratory of the Department of Experimental Physics of the Ivan Franko National University of Lviv. The study was performed by using optical monochromators MDR-2 and MDR-12. The excitation of the blood serum was performed with light with a wavelength of 280 $\mathrm{nm}$, which corresponds to the glow region of human serum albumin. Results: The main indicators, used for the analysis, were the fluorescence intensity $\left(I_{F}\right)$ and the position of the maximum fluorescence band $\left(\lambda_{\max }\right)$. The object of the study was samples of the blood serum of patients of the main and control groups. In the case of the presence of endogenous intoxication in the body, albumin binding centers are blocked by the products of bacterial metabolism and therefore such "pathological" albumin is unable to perform its functions, including transport and detoxification. Conclusions: The pathogenetic concept as the basis of the diagnostic and treating model of purulent-inflammatory diseases and sepsis was devised. In particular, the important role of albumin solution infusion in the treatment of the above-mentioned diseases was established.
\end{abstract}

\section{Keywords}

Purulent-Inflammatory Diseases, Sepsis, Burn Injury, 
Method of Fluorescent Spectroscopy, Albumin Infusion

\section{Introduction}

The fundamental problem of modern medical science remains a significant improvement in the immediate and long-term functional and cosmetic results of surgical treatment of patients. Early detection of sources of infection and effective treatment can significantly help to prevent the development of sepsis in patients. Particular attention should be paid to the problems associated with burns. Their solution will be able to play a key role in clarifying and understanding the main processes occurring in the human body in patients with sepsis. The fundamentally important point is the restoration of the skin in a short time after injury, when patients are not yet exhausted by the healing process, and the regenerative properties of their body are still preserved. Therefore, the objective requirement of the time is to develop new modern approaches in this field of medical science, which are associated with the successful development of biomedical research. The use of the method of fluorescence spectroscopy in medical practice is especially promising.

Pathogenesis is the mechanism of the origin and development of diseases and their individual, including specific, manifestations. It can be seen very carefully at different levels: from noticeable changes at the molecular level to possible disturbances in the body as a whole. Based on the obtained results of the study of pathogenesis, it is possible to assess properly the prognosis of the disease and prescribe a reasonable treatment. It is fundamentally important to develop a scientifically sound algorithm for diagnosing diseases and the effective treatment strategy. This makes it possible to develop the unique algorithm for the diagnostics of their diseases and a full-fledged treating strategy.

The understanding of the pathogenesis of sepsis is the key point in finding effective approaches of its prevention and treatment. It develops when the body's response to the infection causes damage to its own organs and tissues and can lead to significant deterioration of a patient's health or even to death [1] [2]. The antigen in burn shock is tissue proteins, destroyed by a thermal agent, which leads to the synthesis of pro-inflammatory cytokines.

Recovery of affected skin with deep and large burns is a difficult problem. Therefore, even with a favorable course of burn disease, this process lasts at least 1 - 2 months after injury. Through the surface of the burn wound there is a loss of water, proteins, electrolytes, and the wound itself remains a source of infection and intoxication and is the main driving force of pathological changes, that occur in the human body. Therefore, the important component of treatment is the need for rapid and safe removal of necrotic tissue, the use of modern methods of antibacterial therapy, timely correction of metabolic processes and the fastest recovery of lost skin. Therefore, in addition to infusion therapy and sur- 
gical treatment, the use of skin substitutes for temporary closure of burn wounds is an important area of treatment. An important point is also the choice of the optimal time of recovery of the skin in a short time after injury, when patients are not yet exhausted by a long treatment process, and the regenerative properties of the body are still preserved.

\section{Literature Review}

In recent years, a lot of attention was paid to improve the diagnostics and treatment of patients with burn injuries. A particular emphasis should be placed on the feasibility of early surgical treatment with the proper restoration of skin integrity after a burn injury. It is characterized with significant volumes of damaged tissues, which should be eliminated, the activation of cellular and humoral phases of nonspecific resistance and the persistence of microflora. Note, that following endogenous intoxication which occurs in this disease, tissue repair in the area of inflammation and restoration of homeostasis is enormously complicated [3] [4]. Early surgical interventions for the prevention and treatment of wound infection, the restoration of anatomical structures and their rehabilitation are widely used. A separate domain is the study of a wound process and the impact of various drugs on it, as well as that of determining the optimal time of the plastic closure of wound defects [5] [6] [7].

But insufficent attention was paid to the problem of complications after burns, i.e. sepsis. The changes, occuring in patients with sepsis at the molecular level, are understood poorly. At the same time, they play a key role in understanding the processes, occurring in the human body during sepsis and allow the appointment of effective treatment. This problem is very relevant and needs solving.

At the same time, significant progress was made in recent years in the field of combustiology [8] [9]. The concept of early surgical necrectomies of burn wounds with their primary plasticity is widespread. Means of prevention and treatment of wound infection, restoration of anatomical structures and non-surgical correction in the postoperative period are also being developed. Methods of medical and social rehabilitation of patients are also being improved. Considerable attention was also paid to the problems of the functional state of the internal organs, increasing the body's immunoreactivity and combating wound infection.

The authors [10] [11] proposed a method of collection, cryopreservation and lyophilization of xenografts made of pig skin. Lyophilized xenografts are included in the State Register of Medical Devices and approved for use in medical practice in Ukraine in accordance with the Order of the Ministry of Health of Ukraine from 11 May 1998 №115. It is a mandatory element of wound treatment, the purpose of which is to clean it, remove fragments of necrotic formations, disinfect the skin around the wound, wash the wound surface with antiseptic solutions and apply an aseptic dressing. Today drugs based on silver in the treatment of burns are widely used. A technique for saturating lyophilized xe- 
noimplants with silver nanocrystals and using them to treat burn wounds was developed [12].

In patients with superficial burns, silver-rich lyophilized xenoimplants are used to close burn wounds after thorough cleaning under general anesthesia. Wound cleaning is a mandatory element of their treatment, the purpose of which is to remove fragments of necrotic formations, disinfect the skin around the wound, wash the wound surface with antiseptic solutions and apply an aseptic bandage. In patients with burns, lyophilized xenoimplants saturated with silver nanocrystals remain on the wounds for up to $8-10$ days after applying them to them. This eliminates the need for painful dressings. Wound epithelialization occurs directly under lyophilized xenoimplants. Freeze-dried lyophilized xenoimplants adsorb toxins from the wound surface, reduce the inflammatory process in the wound and revascularize areas of necrosis. Later, lyophilized xenoimplants fall off on their own after wound healing. To date, the clinical effectiveness of the use of xenoimplants saturated with silver nanocrystals in the treatment of patients with burn injuries has been proven.

In the treatment of deep burns in the traditional way, local treatment of burn wounds is aimed at restoring microcirculation, creating antibacterial protection and stimulating reparative processes. After chemical necrectomy and wound cleansing, autodermoplasty should be performed. Wounds that are not covered with autodermografts should be closed with lyophilized xenoimplants rich in silver, which temporarily close the wounds. This reduces pain, water, protein and electrolyte loss from the wound, prevents infection and promotes marginal and islet epithelialization. Under the removed xenoimplants there are pure granular granulations, which are ready to accept autografts. Along with the formation of granulation tissue is an active course of epithelialization of the wound surface.

Deep burns with an area of more than $20 \%$ of the body surface often have the following complications: sepsis, erosions and ulcers of the gastrointestinal tract. Immunosuppression, suppression of the cellular immune system and intoxication of the body are also characteristic. Therefore, excision of necrotic tissue significantly improves the patient's condition due to the elimination of the source of infection and intoxication. An open wound surface of a large area leads to depletion of the compensatory capabilities of the human body. Therefore, the technique of early necrectomy with xenodermoplasty prevents progressive intoxication from the affected areas and the development of infection in wounds, reduces the possibility of further progression of burns and leads to the restoration of the skin in the shortest possible time.

Serum albumin composes $60 \%$ of total plasma proteins. It plays an important role in maintaining oncotic pressure. It is also involved in metabolic processes and transports of various chemicals. Albumin is a polypeptide, which consists of 585 amino acids with molecular weight 66.000 - 69.000 Da. Albumin synthesis occurs exclusively in the liver. This provides regular replenishment of albumin 
in the body, which is regulated by osmoreceptors and is inversely proportional to the level of colloid-oncotic pressure.

The detoxification function of albumin is important. Due to the changes of the conformation of its molecules, albumin interacts with hydrophobic molecules of endotoxins and promotes their excretion from the body. Albumin synthesis may decrease under the influence of infusion of synthetic colloids and albumin. It is also slowed by the influence of stress, sepsis, starvation, hyperthermia, and in elderly people. Hormones insulin, cortisone, testosterone, ACTH, growth factors and thyroid hormone can increase the rate of albumin synthesis by hepatocytes. The half-life of endogenous albumin is 21 days, and that of exogenous albumin is 12 hours. Albumin is mainly an extracellular protein. $40 \%$ of albumin is contained in blood plasma and $60 \%$ in the depo (40\% in the skin, $20 \%$ in the muscles of the internal organs) [13]. Albumin enters the interstitium through pores in the endothelium of the capillars and returns to the bloodstream along with the lymph flow. One cycle lasts 15 - 18 hours.

Albumin molecules are able to complex. Diseases, which are accompanied by endogenous intoxication, are characterized with impaired ability of albumin to perform its functions, because some of its binding centers are blocked by toxins. This leads to the deterioration of detoxification capabilities of the body. Although the total concentration of albumin in the body may be within normal limits, its actual "effective" concentration is much lower. Note, that the determination of the "effective" concentration of albumin cannot be performed by modern conventional diagnostic methods, which are currently widely used in the laboratories of health care facilities. Because of the pathogenetic changes of albumin molecules in patients with endogenous intoxication, the effective component of pathogenetic treatment is the use in complex therapy infusions of albumin solution. As mentioned above, the infusion of albumin solution reduces the synthesis of endogenous albumin, but without this infusion, the body will not be able to overcome the infection in case of the presence of endogenous intoxication.

The aim of the research is to create a pathogenetic concept as the basis of a diagnostic and treatment model of purulent-inflammatory diseases and sepsis.

\section{Data and Methology}

Spectral analysis is one of the most important methods for studying the structure of matter and physical processes, which take place in it, including at the molecular level. Luminescence occurs due to the absorption of light by the system under study due to the transition of its molecules from the excited state to the ground state. The absorption and radiation processes are presented in more details in Figure 1. In the initial state (it is also called the ground, unexcited state), the molecule (atom) occupies the energy position with the lowest energy (Figure 1(a)). The absorption of light transfers the system from the ground state to the excited states, which, along with the electron state, also have vibrational energy 
(a)
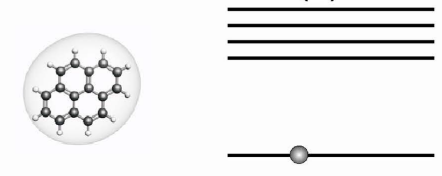

(b)
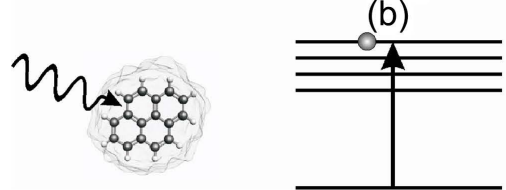

(c)
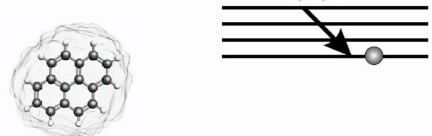

(d)

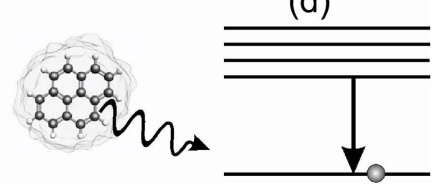

Figure 1. Scheme of luminescence of molecule in the case of irradiation with light.

due to the oscillation of molecules. Therefore, such states are also called electron-oscillating (Figure 1(b)). Due to the oscillating motion, the electronic energy of the molecule turns into oscillating; the molecule loses its energy and for about $10-12$ seconds relaxes to the lowest excited state (Figure 1(c)). The molecule transitions from this lowest excited state to the ground state are accompanied by radiation-luminescence (Figure $1(\mathrm{~d})$ ).

According to the afterglow duration, $\tau$ luminescence is divided into two types: fluorescence if $\tau<10^{-7}$ seconds, i.e. the extinction of luminescence occurs very quickly (for the eye, instantly); phosphorescence if $\tau>10^{-4}$ seconds. In this case, the extinction occurs relatively slowly and is often clearly visible to the naked eye). As part of our own research, we studied radiation with short attenuation times that is fluorescence.

\subsection{Data Source}

In the framework of this research we used general blood test, general analysis of urine, biochemical blood test, bacterioscopic examination, ultrasonographic examination and the method of fluorescence spectroscopy (MFS). The fundamentally important advantages of the MFS are its simplicity, expressiveness, high sensitivity and accuracy, as well as its ability to control effectively biological objects and environments. Thus, the use of this method is very promising for improving diagnostics in medical practice. Within the framework of MFS, it is possible to study the spectral-fluorescent characteristics of biological objects both in normal and in various pathological conditions. In medical practice since 2000, we have been using this method to study the blood serum (BS) and urine of patients, including those with purulent septic complications and sepsis. The study included several stages.

At the first stage, the behavior of the spectral-fluorescent characteristics of the BS of 100 surgical patients with purulent-inflammatory diseases and sepsis and 40 donors were studied. The clinical basis of the study at this stage was the purulent-septic center of Lviv's municipal clinical hospital of emergency medical 
services (Ukraine). The experimental base of luminescent research was the luminescence laboratory of the Department of Experimental Physics of the Ivan Franko National University of Lviv. The study was performed by using optical monochromators MDR-2 and MDR-12. As a source of exciting light, a deuterium lamp DDS-400 with a continuous radiation spectrum in the region $\lambda=200$ - $420 \mathrm{~nm}$ was used. The excitation of the BS was performed with light with a wavelength of $280 \mathrm{~nm}$, which corresponds to the glow region of human serum albumin. The main indicators, used for the analysis of fluorescence spectra of BS, were the fluorescence intensity $\left(I_{F}\right)$ and the position of the maximum fluorescence band $\left(\lambda_{\max }\right)$. BS of patients is a mixture of normal (concentration $\left.X\right)$ and blocked by toxins (concentration 1-X) molecules of albumin. In this case, $\Delta E_{a}$ (Figure 1(d)) is the difference between the energies of the excited and ground states of normal albumin molecules, and $\Delta E_{p}$ respectively, for toxin-blocked albumin molecules. In this case, $\Delta E_{a} \geq \Delta E_{t}$

\subsection{Research Results}

For purulent-inflammatory diseases, three characteristic types of changes of the spectral-fluorescent characteristics of BS were identified, which correspond to aseptic, preseptic and septic pathologies [14]. Figure 2 and Table 1 represent the results of the study of the fluorescence spectra of the BS of the donor and the patient with severe sepsis, caused by purulent epiduritis of the lumbosacral spine and massive

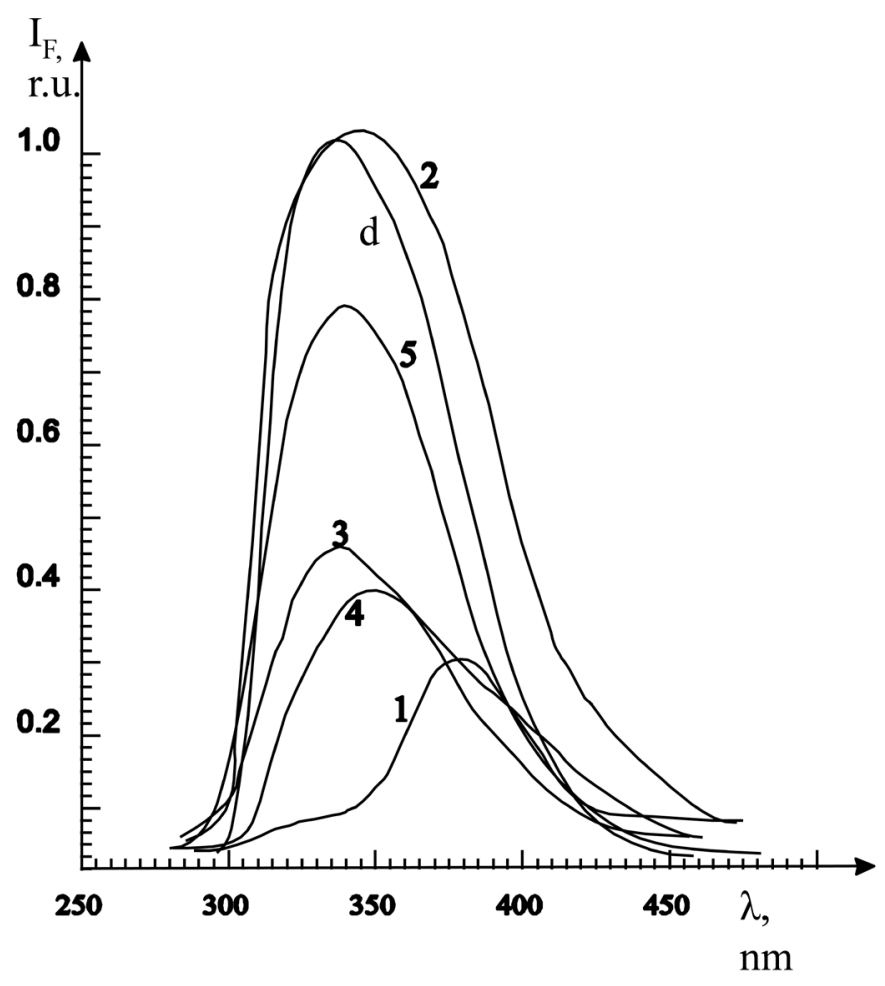

Figure 2. Fluorescence spectra of blood serum of septic patient: 1-28.12.; 2-04.01.; 3-12.02.; 4-19.03. 5-04.06. and donor of BS (340 nm-"normal peak", 380 $\mathrm{nm}$-“septic peak”). 
retroperitoneal intrapelvic phlegmon. She was treated in the hospital from 28 December 2001 till 15 April 2002. At the time of hospitalization (28 December 2001) she was in a critically serious condition with verified bacteraemia (blood culture of 28 December 2001-Staphylococcus aureus). This figure shows that the maximum of the fluorescence band of the patient's BS is shifted to the long-wavelength region by $\Delta \lambda=40 \mathrm{~nm}$ (curve 1) relative to the fluorescence band of the donor, and the fluorescence intensity was $0.3{ }^{*} I_{F}$ of the donor's BS. This contribution is connected with the glow of albumin molecules, blocked by toxins. At the same time, in the region of $330 \mathrm{~nm}$, luminescence intensity due to the contribution of full-fledged albumin molecules is very small. This indicates that the predominant contribution to the intensity of the BS of this patient in a serious condition is made by pathological albumin molecules. The obtained result for the spectral-fluorescent characteristics of the BS at this time indicates a severe septic condition of the patient.

Note, that the survival of the patients in such a serious condition is possible only if $X \geq X^{*}\left(X^{*}-\right.$ is the limit value of the concentration of complete albumin, enough to ensure the survival of patients with sepsis).

After surgical treatment and intensive antiseptic therapy and ongoing bacteraemia (blood culture of 4 January 2002-Staphylococcus aureus), a significant improvement and stabilization of the patient's condition was noted: analysis of the fluorescence spectra of the patient's BS on the seventh postoperative day revealed that the shift of her fluorescence band changed significantly and was $\Delta \lambda=7 \mathrm{~nm}$ (Figure 2, curve 2). At the same time, the intensity of the patient's fluorescence band increased significantly and quite unexpectedly to $1.07{ }^{\star} I_{F}$. Because of subcompensated changes in the absolute quantitative and qualitative content of BS proteins at the time of examination (biochemical studies on 2-4 January 2002: total protein and protein fractions were at the lower limit of nor$\mathrm{mal}$ ), the rapid increase in the fluorescence band of the BS of patient in this case cannot be interpreted by absolute hypoproteinemia that typically causes a weakening of the concentration quenching of fluorescence, which is characteristic of protein fluorescence. The only possible explanation for the phenomenon of increasing fluorescence band intensity of the BS of this patient registered above may be the presence of transient hypervolemia during this period of treatment: the volume of daily intravenous infusions during this treatment period was 8 10 liters.

Under such circumstances, a natural increase in the fluid component of the BS leads to pseudohypoproteinemia-a laboratory phenomenon which is not a

Table 1. Changes of the spectral-fluorescent characteristics of the patient 1 with sepsis.

\begin{tabular}{ccccccc}
\hline $\mathrm{N}$ & $\mathrm{d}$ & 1 & 2 & 3 & 4 & 5 \\
\hline Date & 28.12 & 28.12 & 04.01 & 12.02 & 19.03 & 04.06 \\
$\lambda_{\max }$ & 340 & 380 & 345 & 337 & 349 & 340 \\
$I_{F}$ & 1.0 & 0.3 & 1.07 & 0.46 & 0.39 & 0.79 \\
\hline
\end{tabular}


standard biuret reaction and can be differentiated from true hypoproteinemia only by special techniques and the normogram of Phillips and van Slyke [14] [15].

Note, that the detected changes in the spectral-fluorescent characteristics of BS in patients with sepsis in most cases were pre-manifest: they were usually recorded $24-48$ hours before the appearance of obvious clinical and laboratory signs of significant change in the general somatic status of patients.

Further studies of the fluorescence spectra of this patient showed, that bacteremia was not overcome. Although (see Figure 2, curves 3,4) the long-wave septic peak disappeared only a further long process of treatment under the influence of complex therapy led to a significant suppression of bacteremia and a significant improvement in the patient's condition (Figure 2, curve 5) and she was discharged from the hospital in satisfactory condition.

In our opinion, the forced excessive therapeutic dilution of the blood during this period caused a weakening of the concentration quenching of the fluorescence of the BS of this patient and caused an increase in the intensity of the fluorescence bands of her BS. Undoubtedly, the above-mentioned significant increase in the intensity of the fluorescence band of her BS from 4 January 2002 was influenced by the weakening of septic symptoms.

Thus, according to our studies of the fluorescence spectra of the BS of the above patient, note, that the decrease in intensity and shift of the fluorescence band are due to the presence of advanced septic process and correlate with integrated indicators of clinical severity and bacteremia. The dynamics of changes in the spectral-fluorescent characteristics of the BS of the mentioned patient quite objectively reflects the course of sepsis and correlates with the effectiveness of treatment tactics.

Note, that for the first time we obtained fundamental results for the fluorescence spectra of a patient with sepsis and studied the dynamics of their changes during her recovery [15] [16]. They became a reference point for our further studies of the fluorescence spectra of BS of patients with purulent-inflammatory diseases and sepsis. Note, that the results for the spectral-fluorescence characteristics of two more patients with sepsis for convenience, we will discuss later during the analysis of the corresponding results, obtained by us for patients with burns.

The dilution of BS with distilled water causes an increase of the fluorescence intensity of BS (Figure 3). Note, that the dilution of BS with a solution of albumin has little effect on the spectral-fluorescence characteristics of these solutions. However, the position of the fluorescence bands does not change. Changes in fluorescence spectra during the dilution of BS with distilled water have a specific character and form the basis for the development of the fluorescent method to diagnose various diseases accompanied by hypoproteinemia and hypoalbuminemia and various treatments (study of the effects of infusion therapy). This made it possible to model the effect of infusion therapy on the spectral-fluorescence characteristics of BS of patients. 

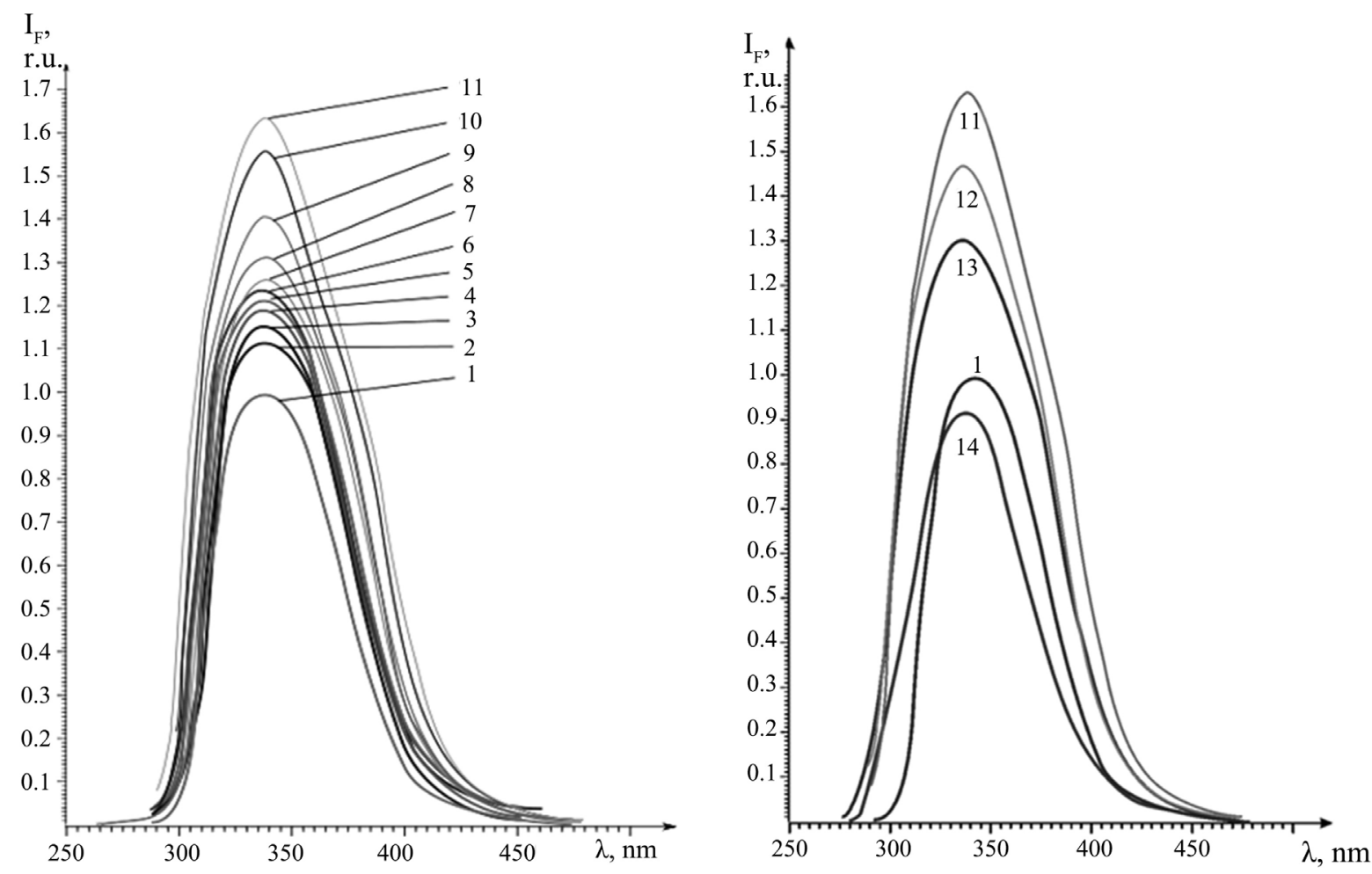

Figure 3. Effect of dilution with distilled water (DW) on the fluorescence spectra of donor blood serum (BS) (1-BS $2-90 \%$ BS, 3-80\% BS, 4-70\% BS, 5-60\% BS, 6-50\% BS, 7-40\% BS, 8-30\% BS, $20 \%$ BS, $10-10 \%$ BS, $11-5 \%$ BS, 12-DW: $\left.I_{F}=0\right)$.

Our in vitro studies of the spectral-fluorescence characteristics of standard dilutions of the donor BS with distilled water (see Figure 3) confirmed the correctness of our proposed explanation of the registered phenomenon of fluorescence band increase in the BS of a patient with sepsis (Figure 2, curve 2).

After all, the decrease in the content of BS in the samples after the addition of distilled water also leads to a significant increase in the intensity of the fluorescence bands.

Dilution of BS by bacterial culture of Staphylococcus aureus (Figure 4, Figure 5) causes gradually decreases of $I_{F}$ with increasing content of bacterial culture in solution. There is also a long-wavelength shift of the fluorescence bands $\left(\lambda_{\max }\right)$ of these dilutions [17]. Note, that the detected effect of changing the spectral-fluorescent characteristics of dilutions of BS by bacterial culture is due to the influence of bacteria and products of their metabolism on the molecules of serum albumin. Note, that changes of the fluorescence spectra of BS during dilution of BS by bacterial cultures have a specific character (Figure 4, Figure 5) and form the basis for the development of the fluorescent method for early diagnosis of sepsis by studying the spectral-fluorescent model of sepsis in vivo [16]. The behavior of the spectral-fluorescent characteristics of dilutions of BS with sugar broth has similar character.

At the fourth stage of the study, the main task was to develop the optimal treatment tactics and methods of effective control of the treatment process for 


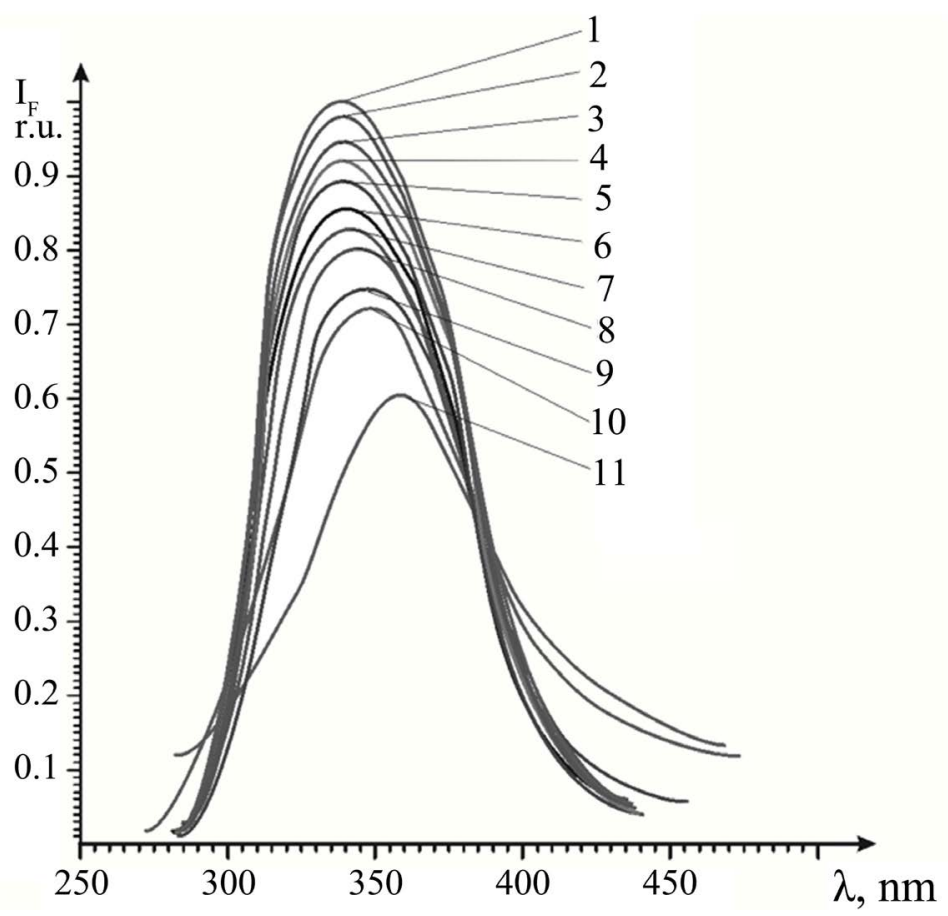

Figure 4. Effect of dilution non-centrifuged (NCF) crops on fluorescence spectra of donor blood serum (BS) (1-blood serum (BS) 2-90\% BS 3-80\% BS, 4-70\% BS, 5-60\% BS, 6-50\% BS, 7-40\% BS, 8-30\% BS, 9-20\% BS, 10-10\% BS, $11-\mathrm{NCF}$ crops). $\lambda_{e x}=$ $280 \mathrm{~nm}$.

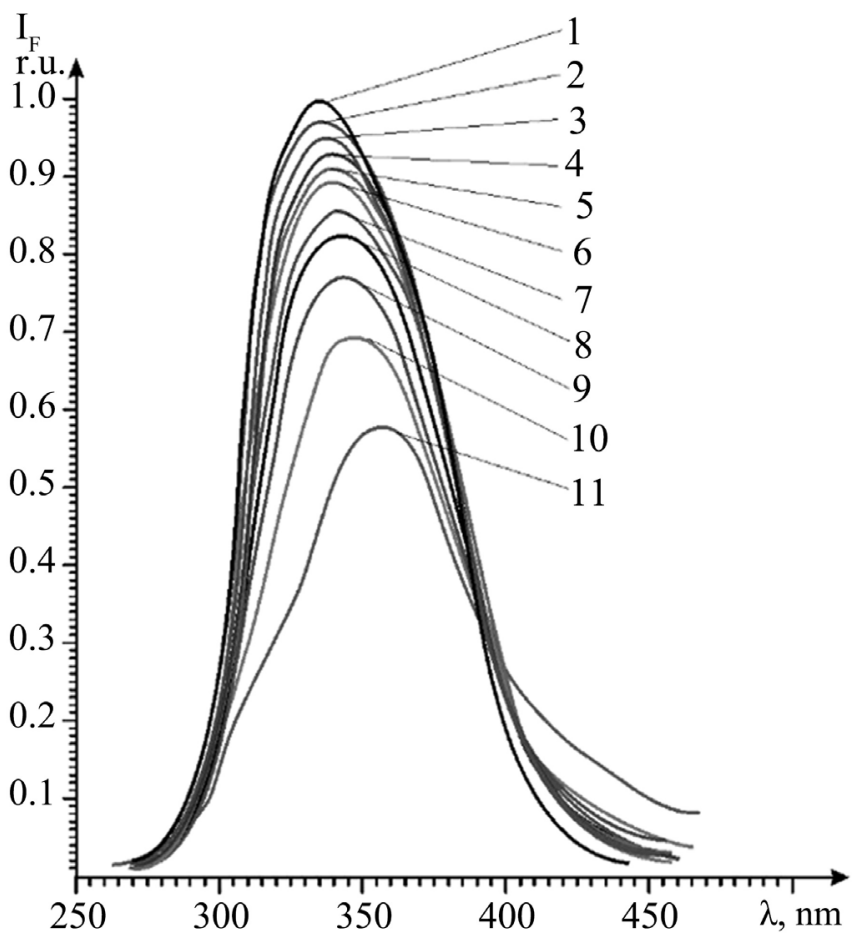

Figure 5. Effect of dilution centrifuged (CF) crops on fluorescence spectra of donor blood serum (BS) (1-blood serum (BS) 2-90\% BS 3-80\% BS, 4-70\% BS, 5-60\% BS, $6-50 \%$ BS, 7-40\% BS, 8-30\% BS, 9-20\% BS, 10-10\% BS, 11-CF crops). $\lambda_{e x}=280$ $\mathrm{nm}$. 
the patients with burn injuries. The clinical base of the study was the burn department of Lviv Communal City Clinical Hospital No 8. The research lasted in 2015-2019.

The main study group consisted of 20 patients. The criteria for selecting patients for follow-up were the presence of superficial and deep burns flame and boiling water burns, including head and neck burns. In the study, we included patients mostly without comorbidities aged 20 - 60 years for the purity of the experiment, because the presence of these comorbidities can dramatically change the behavior of spectral fluorescence parameters of serum.

The control group consisted of 25 healthy individuals (donors) without chronic diseases. For patients and donors within the MFS, a study of samples of their BS was conducted. The comparison group consisted of 25 patients in serious condition, whose BS was not tested by using MFS. But therapeutic tactics with donor albumin solution was also used for these patients. In this study, the classification of burns depending on the etiological factor, depth, area and location of the lesion was used for both groups of patients. The data of laboratory examinations (general analysis of blood and urine, biochemical analysis of blood, bacterioscopic examination) and the results of instrumental methods of examination were analyzed. In the course of the research, we also discussed clinical and anamnestic data and analyzed the spectral-fluorescent characteristics of the BS of patients of the main group, obtained dynamically within the framework of MFS. All the patients in the hospital underwent the appropriate surgical treatment of affected burn surfaces, followed by wound closure with lyophilized xenografts. The wounds were epithelialized partly under dry skin, partly under dry necrosis and applicators. Residual wounds were epithelialized under dry applicators. Patients also received anti-inflammatory treatment, antibiotic and infusion therapy, including with the use of albumin solution and desensitizing therapy. The main regularities of the behavior of the spectral-fluorescent characteristics of the BS of patients with burn trauma were studied and the problems of their treatment were discussed [18] [19] [20] [21]. However, at the same time, the problem of purulent-septic complications in patients with burns was not discussed.

Now we shall discuss the results of the study of the spectral-fluorescent characteristics of the BS of patients with burn injury with septic complications. Figure 6 presents the results of the research in the dynamics of fluorescence spectra and Table 2 contains data for the spectral-fluorescent characteristics of the BS of the patient with burn injury with the area of the burn surface $38 \%$, admitted to the hospital on 27 June 2015.

He was immediately prescribed appropriate treatment, including antibiotic therapy and infusion therapy with the volume of $2-3$ liters daily. Staphyloccus aureus $10^{5}$ and Pseudomonas aeruginosa $10^{6}$ were verified on the basis of a microbiological study. Due to the infusion therapy, the fluorescence intensity of BS during the first 6 days did not decrease significantly $\left(I_{F}=0.88\right.$ r.u.), that correlated with the results of in vitro studies. There was also no shift in the fluorescence 


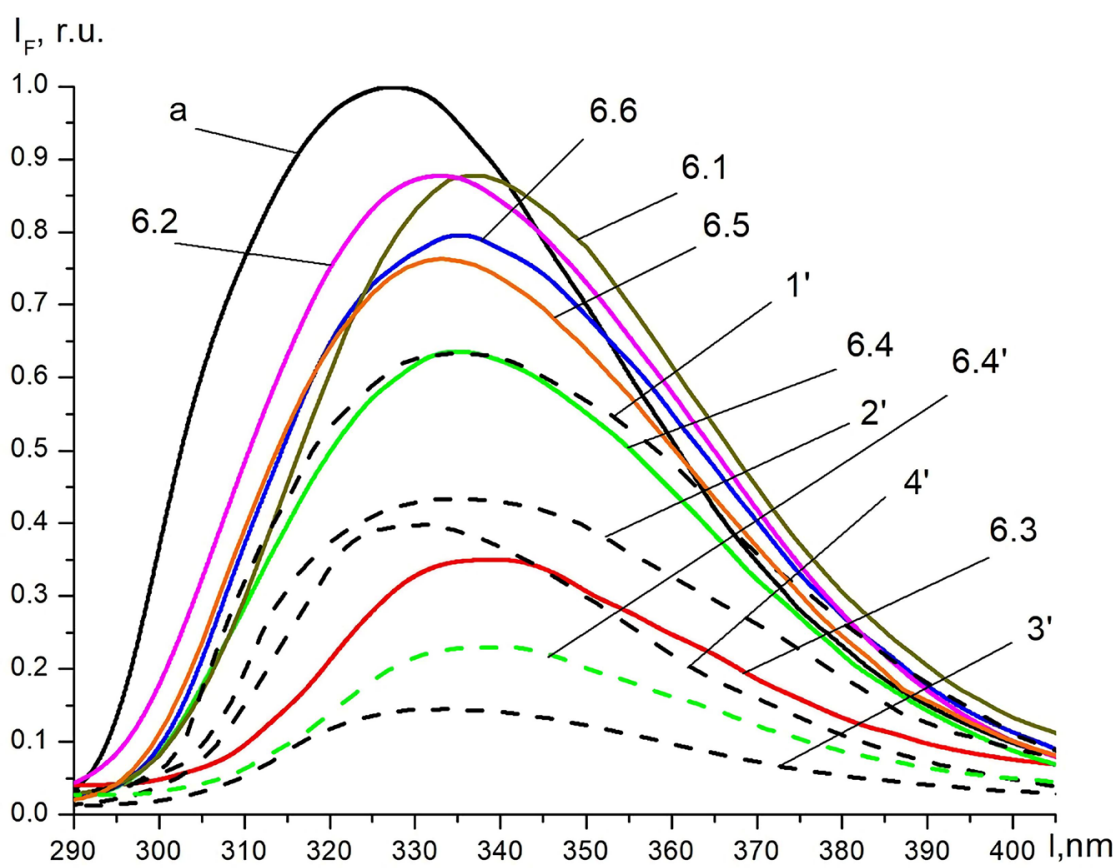

Figure 6. FS of BS of patient 6 with a burn injury, who was hospitalized in Communal City Clinical Hospital №8, Lviv in 2015 in dynamics during treatment (6.1-3.07., 6.2-8.07., 6.3-13.07., 6.4-17.07., 6.4'-17.07., 6.5-20.07., 6.6-24.07.) and a patient with sepsis, who was treated in 2002 in Ambulance hospital (1'-03.06., 2'-05.06., 3'-06.06., 4'-07.06., 5'-10.06) and 20\% albumin solution (a), $\lambda_{e x}=280 \mathrm{~nm}$.

Table 2. Changes of the spectral-fluorescent characteristics of the patient 6 with burn injury.

\begin{tabular}{cccccccccccccc}
\hline № & a & 6.1 & 6.2 & 6.3 & 6.4 & $6.4^{\prime}$ & 6.5 & 6.6 & $1^{\prime}$ & $2^{\prime}$ & $3^{\prime}$ & $4^{\prime}$ & $5^{\prime}$ \\
\hline Date & 3.07 & 3.07 & 8.07 & 13.07 & 17.07 & 17.07 & 20.07 & 24.07 & 3.06 & 5.06 & 6.06 & 7.06 & 10.06 \\
$\lambda_{\max }$ & 327 & 336.1 & 332.2 & 341.1 & 335.1 & 341.1 & 333.1 & 335.1 & 335.2 & 335.2 & 334.1 & 331.6 & 331 \\
$I_{F}$ & 1 & 0.88 & 0.88 & 0.35 & 0.64 & 0.27 & 0.76 & 0.80 & 0.63 & 0.43 & 0.14 & 0.4 & 0.76 \\
\hline
\end{tabular}

spectra of the BS in the long-wavelength region, despite the verification of two pathogens in the patient. The examination of the fluorescence spectra of the BS of this patient on 13 July 2015 (Figure 6, curve 6.3) showed a significant decrease in its $I_{F}$ to 0.35 r.u. and a shift of the fluorescence band in the long-wavelength region by $9 \mathrm{~nm}$. The deterioration of this patient's clinical condition was also revealed. This indicated the deepening of endogenous intoxication in him.

The treatment process was corrected by using the infusion of $20 \%$ donor albumin solution (100 $\mathrm{ml} 8$ times on different days). Subsequent sampling of BS revealed a gradual normalization of the spectral-fluorescent characteristics of the BS of the patient (see Table 2). Therefore, he was discharged from the hospital in a satisfactory condition on 24 of July 2015. Figure 6 shows that the spectral-fluorescence characteristics of this patient are qualitatively correlated with the corresponding results of the patient with sepsis, presented in the same figure 
by dashed curves (curves 1' - 5'). This patient was treated in the hospital in 2002. In order to correct the treatment process of a patient with a burn injury, information about the behavior of the spectral-fluorescent characteristics of a patient with sepsis played a significant role (Figure 6). Without the correction of the treatment process, the condition of the above-mentioned patient with burn injury could continue to deteriorate (Figure 6, curve 6.4') with subsequent transition to a severe septic condition, as it was in case of the patient with sepsis. At the same time, information about the behavior of the spectral-fluorescent characteristics of the patient with sepsis played a significant role in correcting the treatment process of a patient with a burn injury (Figure 6).

The behavior of the spectral-fluorescent characteristics of patients with sepsis, even after burn injuries, is determined by the contributions of two types of albumin molecules: complete and "blocked by toxins". Figure 7 shows, that most of the albumin molecules of a patient with severe sepsis (curve 1) are blocked with toxins (long-wave septic peak). Only a small number of complete molecules of albumin provide support for the vital functions of the patient's body (fluorescence in the region of $330 \mathrm{~nm}$ ). Earlier it was noted, that in such a serious condition of the patient the synthesis of albumin was very slow. However, if this patient (Figure 2) had been given an infusion of $20 \%$ albumin solution, a more noticeable peak in the fluorescence spectra could have appeared in the region of $330 \mathrm{~nm}$ that would lead to the improvement of her condition. But this is only

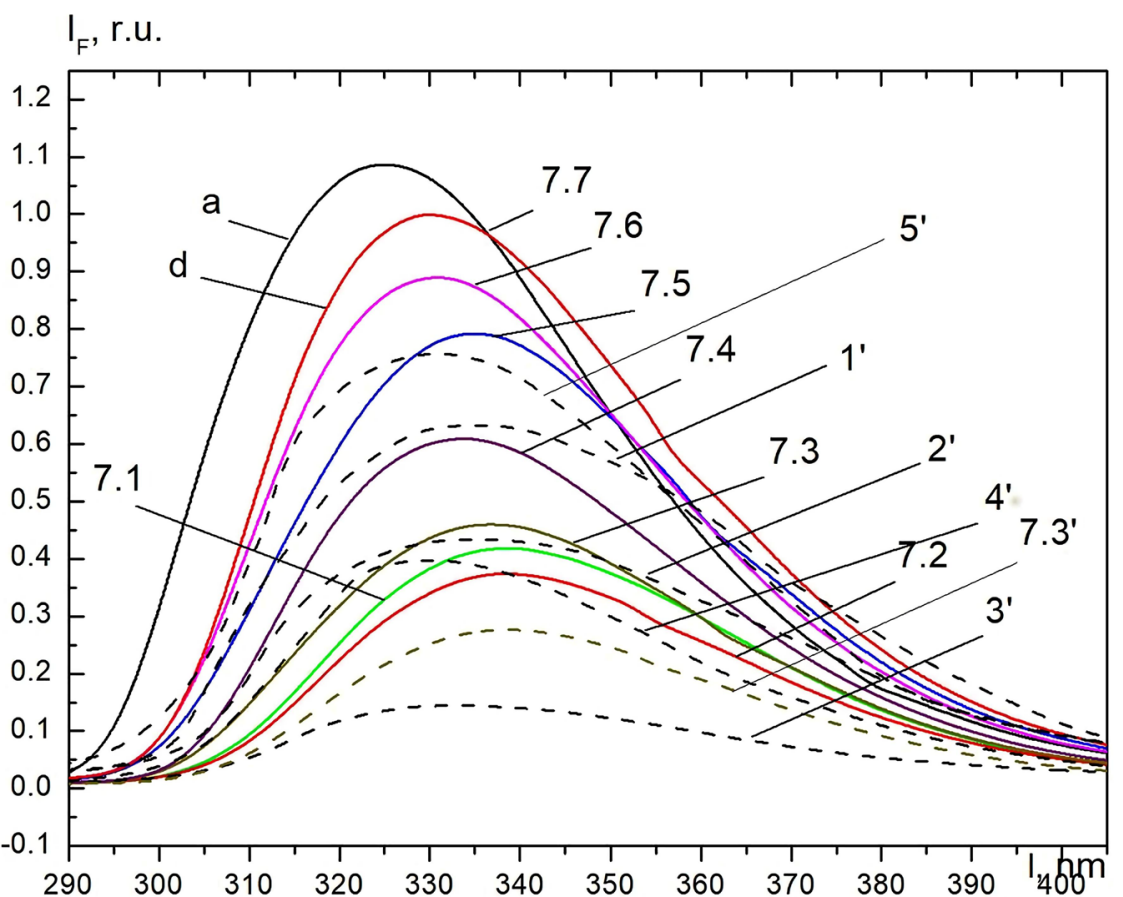

Figure 7. FS of BS of patient 7 with a burn trauma, who was hospitalized in Communal City Clinical Hospital №8, Lviv in 2017 in the dynamics during treatment (7.1-9.02., 7.2-14.02., 7.3-22.02., 7.3'-22.02., 7.4-27.02., 7.5-03.03., 7.6-10.03., 7.7-31.03), and a patient with sepsis, who was treated in 2002 in Ambulance Hospital (1'-3.06. $2^{\prime}-5.06 ; 3^{\prime}-6.06 ; 4^{\prime}-7.06,5$ ' 10.06 ) and $20 \%$ albumin solution (a), $\lambda_{e x}=280 \mathrm{~nm}$. 
our assumption. Strictly speaking about this patient, it was not proven what changes would occur in the fluorescence spectrum during the infusion of $20 \%$ albumin solution. This may be the subject of our further studies of the spectral-fluorescent characteristics of patients with sepsis after a burn injury in such a severe condition.

The results of the research of fluorescence spectra of the patient with the burn injury with the burn surface area 28\%, who was hospitalized in February 2017, in the dynamics, are presented in Figure 7, and the data for the spectral-fluorescent characteristics of his BS are depicted in Table 3.

He was immediately given appropriate treatment, including antibiotic and infusion therapy of up to 3 liters daily, as well as infusions of $10 \%$ donor albumin (February 6 and 10) in amount $100 \mathrm{ml}$. The condition of this patient was much more severe than the previous one. Despite intensive treatment, his condition deteriorated markedly during the first 5 days. This is evidenced by the decrease in fluorescence intensity and a slight long-wavelength shift (Figure 7, curves 7.1, 7.2).

Compared with the previous patient in this case, most likely, there was a more noticeable endogenous intoxication. Therefore, the correction of the treatment process was performed for him, including the infusion of $10 \%$ solution of albumin (February 15, 18, 26 and March 2 in the amount $100-150 \mathrm{ml}$ ).

It is obvious that the intake of a sufficient amount of albumin significantly improved the work of the body's detoxification systems with the subsequent normalization of the body's synthesis of endogenous albumin. As a result, the fluorescence intensity of the patient's BS gradually increased, and the long-wave shift leveled off. After that, the patient was discharged from the hospital in a satisfactory condition. Without correction of the treatment process, the patient's condition could have deteriorated (Figure 7, curve 7.3') towards a subsequent transition to the severe septic condition, as it was as in case of the patient with sepsis.

Our results for the spectral-fluorescence characteristics of the above-mentioned patient with burn injury correlate well with the corresponding results (Figure 7, curves 1' - 5') for a patient with sepsis, treated in hospital in 2002. Regardless of the etiological factors of sepsis, the pathogenetic mechanisms of septic complications are unified. Serum albumin molecules have the ability to complexation. In case of the presence of endogenous intoxication in the body, they are blocked by the products of bacterial metabolism. Understanding the microscopic mechanisms

Table 3. Changes of the spectral-fluorescent characteristics of the patient 7 with burn injury.

\begin{tabular}{cccccccccccccccc}
\hline № & $\mathrm{a}$ & $\mathrm{d}$ & 7.1 & 7.2 & 7.3 & $7.3^{\prime}$ & 7.4 & 7.5 & 7.6 & 7.7 & $1^{\prime}$ & $2^{\prime}$ & $3^{\prime}$ & $4^{\prime}$ & $5^{\prime}$ \\
\hline Date & 9.02 & 9.02 & 9.02 & 14.02 & 22.02 & 22.02 & 27.02 & 03.03 & 10.03 & 31.03 & 3.06 & 5.06 & 6.06 & 7.06 & 10.06 \\
$\lambda_{\max }$ & 330.1 & 333.1 & 335.1 & 339.1 & 337 & 337 & 334 & 335.1 & 331.1 & 332.0 & 335.2 & 335.2 & 334.1 & 331.6 & 331 \\
$I_{F}$ & 1 & 1 & 0.41 & 0.37 & 0.46 & 0.27 & 0.61 & 0.79 & 0.89 & 0.95 & 0.63 & 0.43 & 0.14 & 0.40 & 0.76 \\
\hline
\end{tabular}


of the theory of pathological albumin formation is the basis for the development of pathogenetic treatment tactics.

The most optimal approach for the detection of septic conditions in patients is the study of the spectral-fluorescent characteristics of their BS in the frame of the method of fluorescence spectroscopy. There is a high risk of septic condition in patients with burn injuries in two cases: in patients with the large area and depth of burn trauma and in the case of inadequate treatment at the initial stage of the disease. Therefore, the key thesis of successful treatment is the comprehensive approach to prevent the development of bacteraemia owing to the early surgical treatment and comprehensive therapy. The fundamental idea of successful completion of the treatment process is constant monitoring within the method of fluorescence spectroscopy of the treatment process with the possibility of its correction. In the treatment process, the intensity and position of the maximum fluorescence of the BS of patients are the indicators of fundamental importance. The lower the fluorescence intensity, the higher the probability of exitus letalis.

To illustrate the above considerations, we present the results of the studies of the spectral-fluorescent characteristics of the BS of the patient with sepsis and diabetes mellitus (Figure 8, Table 4), who was treated at the hospital in 2002.

The patient's condition deteriorated steadily during the follow-up period, despite surgery and intensive antibiotic therapy that may be explained by the presence of a number of severe comorbidities and her advanced age. The negative dynamics of this patient's condition is reflected by the unfavorable dynamics of the parameters of the spectral-fluorescent characteristics of her BS: a constant decrease in the intensity of the fluorescence bands (Figure 8, curves 1,2,3).

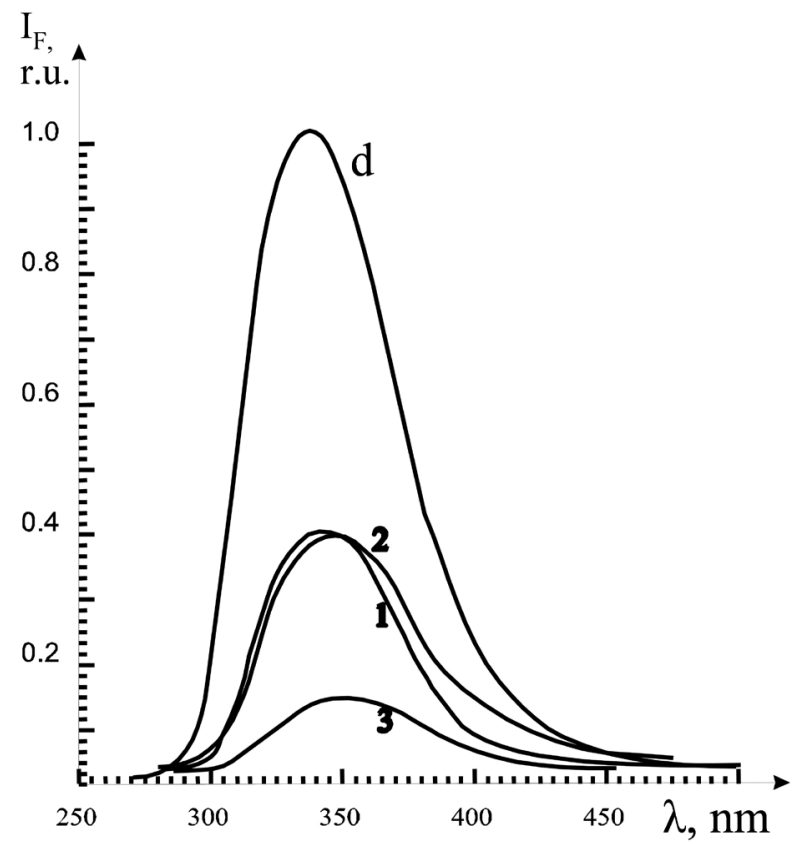

Figure 8. Fluorescence spectra of BS of patient with sepsis and diabetes: 1-03.06; $2-05.06 ; 3-06.06$ and donor BS. $\lambda_{e x}=280 \mathrm{~nm}$. 
Table 4. Changes of the spectral-fluorescent characteristics of the patient with sepsis and diabetes.

\begin{tabular}{ccccc}
\hline $\mathrm{N}$ & $\mathrm{d}$ & 1 & 2 & 3 \\
\hline Date & 03.06 & 03.06 & 05.06 & 06.06 \\
$\lambda_{\max }$ & 338 & 342 & 347 & 351 \\
$I_{F}$ & 1.0 & 0.41 & 0.40 & 0.15 \\
\hline
\end{tabular}

It is logical to assume that it is appropriate to use the infusion of albumin solution to prevent the deterioration of the patient's condition. Obviously, such a treatment procedure should be performed. However, unfortunately, this idea was not implemented in her treatment. We do not have a reliable answer about the possibility of recovery of this patient using the infusion of albumin solution. Unfortunately, the mentioned patient died as a result of the advanced process of the generalization of infection and multiorgan failure. The dynamics of changes in the spectral-fluorescent characteristics of the BS of patients with sepsis objectively reflects the clinical features of the disease, which significantly depends on the quality of diagnosis and correlates with the effectiveness of treatment tactics.

In conclusion, it is important to note that the dynamics of changes in the spectral-fluorescent characteristics of the BS of patients with sepsis during treatment objectively reflects the clinical features of the disease. Successful completion of the treatment process significantly depends on the quality of diagnosis and monitoring and correlates with the effectiveness of treatment tactics. For successful and effective management of the treatment process within the MFS, it is very important to have portable equipment for the study of fluorescence spectra, as well as reliable financial support of the treatment process. If the septic process becomes uncontrollable and cannot be treated, the probability of patient's survival is low. But the use of MFS in the treatment can help to prevent this. There is enough time to adjust the treatment process and help the patient survive. Concomitant diseases that are incompatible with life can become an obstacle to this.

In conclusion, we would like to concentratre on the problems that need to be solved in order to improve properly the treatment process of patients with burn injuries to prevent the development of the septic process. It is necessary to conduct a clinical and laboratory examination of the patient, prescribe antibiotic and infusion therapy, including the use of albumin solution. It is fundamentally important to carry out early surgical treatment to prevent the development of endogenous intoxication of the body. It is important to sow bacteria in order to verify and study their sensitivity to antibiotic therapy. Empirically, it is advisable to prescribe broad-spectrum antibiotics and wait until the results of crops. This algorithm is optimal for the correction of antibiotic therapy. The authors of the research [22] on the basis of a multicentric study demonstrated the effectiveness of the fluorescent method for the diagnosis of bacterial contamination of wounds. The accuracy of this method has been found to be higher than standard 
research methods, which are widely used in the routine practice of health care facilities. In this study, the fluorescent method allowed to change treatment tactics in $69 \%$ of patients and improve the provision of medical services to patients.

In the critical situation, the fluorescence intensity of albumin molecules is of fundamental importance. The most fundamental is the registration in time of the threatening moment which requires a rapid correction of treatment tactics. If the choice of antibiotic was not correct, it is necessary to replace antibiotics in accordance with the results of the determination of sensitivity according to the antibioticogram. In healthy people, up to $6 \%$ (average $4 \%-5.8 \%$ ) of albumin molecules are glycosylated, while in patients with diabetes such molecules are more than $9 \%(9 \%-12 \%)$. The albumin glycosylation of more than $12 \%$ indicates the presence of decompensated diabetes mellitus. This reduces the amount of albumin, which is able to perform a detoxifying function, which slows down the healing process of wounds in patients with diabetes. Such patients have a predisposition to the prolonged course of purulent-septic complications and the likelihood of exitus letalis (Figure 8).

Given our successful experience of using infusion therapy with albumin solution for the treatment of patients of the main group with burn injuries, we applied this experience to the comparison group. Consider in more details several clinical cases of patients from the comparison group in serious condition, which deserve attention. The 38-year-old patient was admitted to the burn department on 29 September, 2018 with superficial and deep flame burns (70\% of the head, torso, both upper and lower extremities) and second-degree burn shock. The daily volume of infusion therapy was $3700-4000 \mathrm{ml}$. The patient also had severe septicotoxemia, according to laboratory examination, mild anemia and hypoproteinemia. The patient received antibacterial, anti-inflammatory therapy and infusion of albumin solution for 6 times (three times - 20\% and 6 times - 10\%). The patient also underwent surgery-necrectomy and free autodermoplasty. During the treatment, the patient's health was closely monitored and treatment tactics was adjusted. After 41 days, the patient was discharged in satisfactory condition under the supervision of a surgeon at the place of residence.

It is necessary to dwell in more detail on the peculiarities of the course of burn injury of another patient, 28 years. The patient was hospitalized in the burn department on 20 June, 2018 with IIB degree gas flames up to $50 \%$ of the surface of the head, neck, torso, upper and lower extremities. The patient also had a first-degree airway burn and a second-degree burn shock. He was hospitalized in serious condition. Patient received antibacterial, antiplatelet therapy. As part of the infusion therapy, he also received infusions of $20 \%$ albumin solution $(100 \mathrm{ml}$ three times). The patient also underwent two surgeries-necrectomy (27 June, 2018) and free autodermoplasty (12 July, 2018). The patient was hospitalized for 40 bed days and was discharged home on 30 July, 2018 in satisfactory condition. Note that the above patients were in a condition very close to septic.

A 38-year-old patient's was treated in the hospital from the 30 August to the 13 November, 2019. At the time of admission, the patient's condition was se- 
rious. The main diagnosis was second-degree (type A and B) flame burn of $35 \%$ of the head, neck, back and both upper limbs, second-degree burn shock. The patient had a fever and endogenous intoxication. The general blood test revealed leukocytosis with the increased number of rod granulocytes and the increased rate of erythrocyte sedimentation. The patient underwent a successful surgical treatment. He received an anti-inflammatory, antibacterial, anticoagulant, antifungal and hormonal therapy, infusions of albumin solution. Total amount of albumin solution was $700 \mathrm{ml}$. The patient also received erythromass (4 times) and native plasma (5 times). The daily infusion volume was more than $3000 \mathrm{ml}$. The patient underwent successful surgical treatment-staged necrectomies, xenoplasty with lyophilized xenoimplants saturated with silver nanocrystals and autodermoplasty. The patient's condition was under reliable monitoring. Infusions of albumin solution were provided in the most critical periods of the patient's condition. They made it possible to balance the amount of complete albumin in the BS and improve the patient's condition. After successful completion of the treatment process (75 bed days), the patient was discharged from the hospital in satisfactory condition.

Thus, this section, based on the results of the study of septic complications of patients with surgical profile, illustrated the successful experience of using MFS to diagnose, control and improve the treatment of patients with burn injuries in a condition close to septic. It is shown, that the experience and skills gained in this case significantly helped to improve treatment tactics in the presence of septic complications in patients with burn injuries. Fundamentally important in the future are thorough studies within the MFS of different scenarios for the development of septic complications in severe forms of burns. The results obtained in our study showed that the method of fluorescent spectroscopy had sensitivity $100 \%$ and specificity $88.0 \%$.

\section{Conclusions}

The pathogenetic concept of diagnostic and treatment model of purulent-inflammatory diseases and sepsis is presented. It is based on the fact, that in diseases, which are accompanied by endogenous intoxication, part of the albumin molecules in the patient's blood is blocked by toxins. As a result, there are two types of albumin molecules in the blood of patients: normal and blocked by toxins. This leads to a deterioration of the detoxification capabilities of the body. For diagnosis, especially early, monitoring and correction of the treatment process, the method of fluorescence spectroscopy is proposed. It has been established that the spectral-fluorescent characteristics of the serum of patients with purulent-septic complications were universal markers of the severity of the patients' condition. The peculiarities of the behavior of these markers for various specific diseases were illustrated. At the same time, some changes of the spectral-fluorescent characteristics of the patients' blood serum were registered 24 48 hours before the emergence of obvious clinical and laboratory signs of the pa- 
tients' general somatic condition. The results obtained with the help of MFS are significantly ahead of the results of other research methods, which are now routinely widely used for diagnosis in health care facilities.

In order to overcome optimally endogenous intoxication, it is proposed to use infusions of albumin solution to increase the content of complete serum albu$\mathrm{min}$ in patients with endogenous intoxication. It is shown, that the scenarios of sepsis, including in the case of burns, depend on the severity of the disease. The features of the pathogenesis of sepsis do not depend on its etiological factors.

\section{Conflicts of Interest}

The authors declare no conflicts of interest regarding the publication of this paper.

\section{References}

[1] Kozynets, G.P., Sorokina, O.Yu., Slesarenko, S.V. and Philip, Zh.V. (2017) Modern Definition of Sepsis and Septic Shock in Patients with Deep Burns. Surgery of Ukraine, 1, 109-117.

[2] Sorokina, O.Y. and Koval, M.G. (2020) Screening and Diagnosis of Sepsis in Patients with Severe Burns. Emergency Medicine, 16, 16-22. https://doi.org/10.22141/2224-0586.16.1.2020.196925

[3] Shano, V.P., Cherny, V.I. and Nesterenko, A.N. (2001) Endotoxicosis, Immune Distress and Multiorgan Disorders: Clinical and Morphological Justification of Therapy from the Standpoint of SIRS. Pain, Anesthesia and Intensive Care, 2, 45-47.

[4] Willis, M.S., Carlson, D.L., DiMaio, J.M., et al. (2005) Macrophage Migration Inhibitory Factor Mediates Late Cardiac Dysfunction after Burn Injury. American Journal of Physiology Heart and Circulatory Physiology, 288, 795-804. https://doi.org/10.1152/ajpheart.00189.2004

[5] Savchyn, V.S. (2014) Features of Reparative Processes in Patients with Deep Burns of the Head and Neck. Archive of Clinical and Experimental Medicine, 23, 149-152.

[6] Boyarska, G.M. (2009) Peculiarities of Changes of Phagocytic Activity of Neutrophilic Granulocytes of Peripheral and Capillary Blood of the Zone of Thermal Damage in Victims of Severe Burns. Clinical Surgery, 11/12, 18.

[7] Kovalenko, O.M. (2014) Questions of Infusion Therapy of Patients with Burn Shock. Surgery of Ukraine, 2, 13-19.

[8] Kovalenko, O.M. (2012) Pathogenetic Substantiation of Programs of Surgical Treatment of Children with Common Burns and Their Influence on the Course of Wound Process. Doctor's Thesis, O. Bohomolets National Medical University, Kyiv, 298.

[9] Nagaichuk, V.I., Kozynets, G.P. and Chornopyschuk, R.M. (2019) Modern Tactics of Surgical Treatment of Patients with Burns. Monograph. Konsol, Vinnytsia, 330 p.

[10] Bigunyak, V.V. and Luchanko, P.I. (1993) Method of Lyophilization of Xenodermografts. Patent of Ukraine No. 10737. Kiev, Ukraine.

[11] Bigunyak, V.V. (1995) Method of Treating Wounds Using Lyophilized Xenodermotransplants Saturated with Silver Nanoparticles. Canned Auto- and Xenografts in the Restoration of Lost Skin in Patients with Burns. Doctor's Thesis, Ukraine, Ternopil, 275.

[12] Savchyn, V.S., Lukavetsky, O.V., Guda, N.V., Stoyanovsky, I.V., Chemeris, O.M., 
Tuzyuk, N.V. and Farmaga, T.I. (2015) Method of Treating Wounds Using Lyophilized Xenodermotransplants Saturated with Silver Nanoparticles. Patent of Ukraine No. 102105. Lviv, League Press.

[13] Dziuba, D.O. and Galushko, O.A. (2016) Albumin and Gelatin Solutions. Acute and Urgent Conditions in the Doctor's Practice, 6, 15-18.

[14] Herych, I.D., Bulavenko, O.V., Ostapiuk, L.R., Voloshinovskii, A.S. and Myagkota, S.V. (2015) Fluorescence Spectroscopy: Possibilities of Application in Medical Practice. Lviv, 366.

[15] Ostapiuk, L. (2019) Diagnostic and Therapeutic Model of Sepsis and Purulent-Inflammatory Diseases. International Journal of Clinical Medicine, 10, 577-595. https://doi.org/10.4236/ijcm.2019.1011047

[16] Herych, I.D., Bulavenko, O.V., Ostapiuk, L.R., Voloshinovskii, A.S. and Myagkota, S.V. (2013) Method for Early Diagnosis of Septic Complications by the Method of Fluorescence Spectroscopy. Patent No. 76953 Ukraine Applicant and Patentee: Pirogov Vinnytsia National Medical University.

[17] Bulavenko, O.V., Herych, I.D., Ostapiuk, L.R., Voloshinovskii, A.S., Myagkota, S.V. and Vashchuk, V.V. (2013) Modelling Changes in Blood Serum at Different Diseases and Therapeutic Measures. Biomedical and Biosocial Anthropology, 20, 8-14.

[18] Savchyn, V.S., Ostapiuk, L.R., Voloshynovskyi, A.S. and Malyi, T.S. (2016) Approbation of the Fluorescence Spectroscopy Method for the Diagnosis of Endogenous Intoxication with Burn Injury. Clinical Surgery, 6, 68-70.

[19] Savchyn, V.S., Ostapiuk, L.R., Voloshynovskyi, A.S. and Malyi, T.S. (2019) A New Look at the Diagnosis of Endogenous Intoxication in Patients with Burn Injury. Journal of Hospital Surgery, 1, 20-24.

[20] Zaporozhan, S., Savchyn, V., Ostapiuk, L., Voloshinovskii, A., Tuziuk, N. and Malyi, T. (2020) The New Approach to the Diagnostics and Treatment of Endogenous Intoxication in Patients with Burn Injury. International Journal of Clinical Medicine, 11, 375-388. https://doi.org/10.4236/ijcm.2020.116033

[21] Zaporozhan, S.Y., Savchyn, V.S., Ostapiuk, L.R. and Tuziuk, N.V. (2020) The New Model of Diagnostics, Treatment and Prevention of Purulent-Septic Complications in Patients with Burn Injury. Challenges of Medical Science and Education: An Experience of EU Countries and Practical Introduction in Ukraine. Collective Monograph, 107-141. https://doi.org/10.30525/978-9934-588-64-8-7

[22] Lam Le et al. (2020) Diagnostic Accuracy of Point-of-Care Fluorescence Imaging for the Detection of Bacterial Burden in Wounds: Results from the 350-Patient Fluorescence Imaging Assessment and Guidance Trial. Advances in Wound Care, 10, 123-136. https://doi.org/10.1089/wound.2020.1272 